\title{
New Algorithm for Fault Diagnosis of Photovoltaic Energy Systems
}

\author{
A.H.Mohamed \\ Solid State and Electronic Accelerators Dept., \\ (NCRRT), Atomic Energy Authority.
}

\begin{abstract}
In the recent years, Artificial Neural Networks (ANNs) have proved their great success for fault diagnosis in many applications. The performance of the ANN depends on its topology. So, optimizing the ANN's topology is an essential task to improve its efficiency. On another side, genetic algorithms (GAs) are becoming a main optimization methodology used in solving the engineering problems. However, the proposed system introduces a new algorithm that uses genetic algorithm to optimize the topology of the ANN. It has been used for diagnosis and repairing the Photovoltaic (PV) energy systems dynamically online as a case of study. Recently, PV systems have a great attention and concerning by the researchers to solve the power problems all over the world. Many diagnostic systems have been developed to diagnose the PV faults, but they are very expensive and sometimes are unmanaged especially for complex and critical PV systems. The proposed system enables the PV systems can overcome these limitations. Its obtained results are compared with fuzzybased and traditional neural network-based diagnostic PV systems. It is found that, the proposed system has proved its goodness for the practical applications.
\end{abstract}

\section{Keywords}

Neural Network, Genetic Algorithm, Photovoltaic Systems, Fault Diagnosis, and Fault Repairing.

\section{INTRODUCTION}

Recently, there is a great power problem appeared all over the world. However, researchers found that using the renewable energy sources represent a key of solution for this problem.

PV systems represented a main area in the renewable energy. They preferred due to their clean and eco-friendly for the environment. They have been successfully used for many applications [1].

The architecture of the target PV system is shown in fig. (1) that consists of a PV panel, a charger and a battery. The panel module is the main part of any PV system. It consists of PV cells connected in a series-parallel NxM array. The output of the panel varies with solar irradiance level and temperature. This drives to use a charger module that controls the PV panel output $\left(\mathrm{V}_{\mathrm{PV}}, \mathrm{I}_{\mathrm{PV}}\right)$ and feeds the power for the load and charging the battery. The battery can store the PV power to feed the needed power for the load in case of unsuitable power obtained from the PV panel. So, the battery is considered an essential part for any PV system [2].

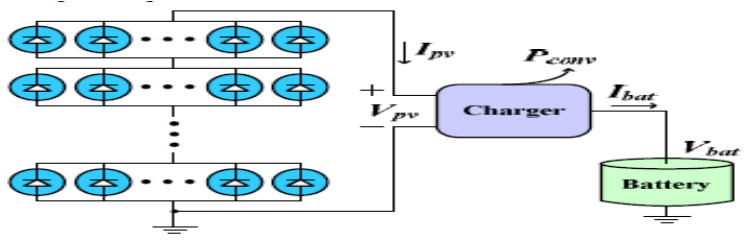

Fig. (1): PV system architecture

\author{
A.M.Nassar \\ Electronic and Communication Dept., \\ Faculty of Engineering, Cairo University
}

But, the efficiency of the PV systems affected by their faults. Ensuring the good performance operation of the PV cells is very important task. Appearing the PV cell faults in the panel causes two problems: (1) A PV cell fault leads to a reverse bias operation which may result in hot spots and cause more faulty PV cells in that PV cell group. (2) limiting the output current of the PV panel [3].

Several PV cell fault detecting and diagnostic techniques have been developed, as: Traditional approaches based on power losses have been proposed for detecting the faults [4-6]. While, a decision tree model and time domain reflectometry (TDR) were used for detecting the faults of PV systems [7-8]. More recently, intelligent systems based on (Neural network, Fuzzy systems or Neuro-Fuzzy network) for automatic detecting the faults in PV fields were introduced [9-11]. Besides, signal processing is used to detect online fault in PV systems [12, 13].

But, these previous techniques suffer from some limitations as: (1) the traditional and decision tree methods were concerning only on the detection of the faults, or sometimes, having no ability to diagnose the causes for these faults, and needing for high cost till achieve the diagnosis [14]. Also, the previous works of the intelligent systems did not develop a complete algorithm and methodology for faults detection and classification that is able to represent the real system effectively [15]. Besides, all these diagnostic system works offline and have not introduced repairing methods for the faulty systems that is considered main goal for the critical systems.

Therefore, the present research proposes a new online optimized neural network fault diagnostic and repairing system. This enables the PV system to be suitable for the critical applications. It can be applied for diagnosis and repairing different types of systems. In this research, it can diagnose and reconfigure the PV panels for failures appeared in a bulletin board in the high way.

The reminder of this paper is organized as follows: Section 2 represents the Neural Networks. Section 3 introduces a short brief about the genetic algorithm. Section 4 presents the proposed system. In Section 5, the applicability of the proposed diagnostic system and their results are given, and the conclusions are addressed in Section 6.

\section{ARTIFICIAL NEURAL NETWORK}

The artificial neural network (ANN) is used mainly to simulate the behavior of nerve system in the human brain. ANN can be represented as parallel distributed processor consists of simple processing units. ANN is simple algorithm, fast computational speed and high accuracy of validation [16]. ANNs have been applied successfully in many fields such as data analysis, fault diagnosis, voice and image recognition, process monitoring, automatic control and optimization resolution [17].

Generally, the ANNs methods have three important stages. They are: data collection, data training (or learning) process and validation of its output. Feed-forward and Backpropagation are the common training algorithms of ANNs [18]. 
There are many different types of network architectures. All of them have three main layers: input, output and hidden layers as shown in fig. (2).

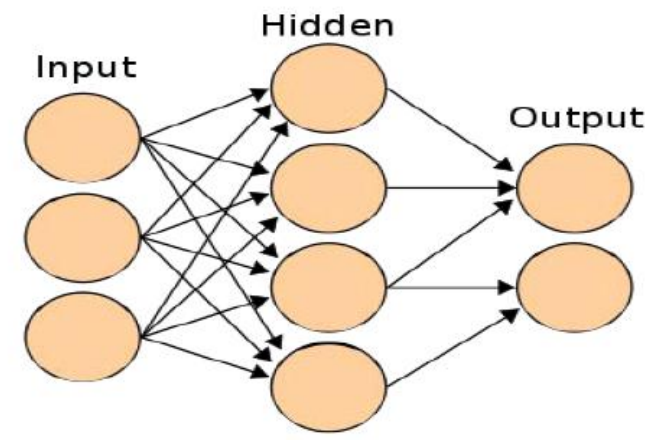

Fig. (2): Structure of Artificial Neural Network

\section{GENETIC ALGORITHMS}

Genetic algorithm (GA) is a global, parallel, stochastic search methods, founded on Darwinian evolutionary principles. They have proved their potential in scientific and engineering optimization or search problems. They can evaluate many solutions in parallel at once. Genetic algorithms are particularly well-suited to solve problems where the space of all potential solutions is truly huge - too vast to search exhaustively in any reasonable amount of time [19].

The GA works with three operators, they are: selection, crossover and mutation. The selection operator determines which individuals may survive and has the chance to continue in the next generation. While, the crossover operator entails choosing two individuals to swap parts of their code, producing new "offspring" that are combinations of their parents. Besides, the mutation operator alters one or more components of a selected chromosome. Mutation randomly changes a gene on a chromosome. It provides the means for introducing new information into the population. Finally, the GA tends to converge on an optimal or near-optimal solution through these operators [20-21].

\section{PROPOSED FAULT DIAGNOSTIC ALGORITHM}

Neural Networks have proved their great success in the diagnostic process. Its performance is mainly depending on its topology. Thus, optimizing the topology of the ANN can ensure the accuracy of its output.

Therefore, firstly, the proposed system uses the genetic algorithm to optimize the topology structure of the neural network that used in the diagnostic process of the PV systems. The genetic algorithm can be implemented as:

1.Chromosome Representation: The proposed system uses the binary digits for representing the topology of networks (chromosomes).

2.Initial Population: The initial population is constructed from random individuals its size $=30$.

3. Crossover Operator: The suggested GA can combine the parents to obtain two offsprings using a uniform crossover. Its rate $=0.2$.

4.Mutation Operator: The random mutation is used for this GA with a rate $=0.03$.
5.Fitness function: Minimizing the error value of the neural network represented the fitness function of the GA. It is computed by the inverse of an error value:

$$
E=100 \frac{O_{\max }-O_{\min }}{n T} \sum_{t=1} \sum_{i=1}\left(y_{i}(\mathrm{t})-z_{i}(\mathrm{t})\right)^{2}
$$

where $\mathrm{t}=1,2, \ldots, \mathrm{T}: \mathrm{T}$ is the number patterns of a validation set containing; $o_{\max }$ and $o_{\min }$ are the maximum and minimum values of output coefficients in the problem representation, $\mathrm{i}=1,2, \ldots, \mathrm{n}: n$ is the number of output nodes, $Y_{i}(t)$ and $Z_{i}(t)$ are actual and desired outputs of node $i$ for pattern $t$.

If $\mathrm{E}$ has not been significantly reduced, then the assumption is that the network is trapped in a local minimum and the network is marked with 'failure'. Otherwise the network is marked with success.

After the optimum topology for the neural network is achieved by the genetic algorithm, secondly, the proposed system divides the structure of PV system into three modules, as shown in fig. (3). They are:

(1) PV panel module contains the master panel that feeds the power for the load and the auxiliary PV cells. Then, the proposed diagnostic system divides the PV panel into multiple areas. Each of them has its inputs and related output for training the neural network.

(2) A charger module.

(3) A battery module.

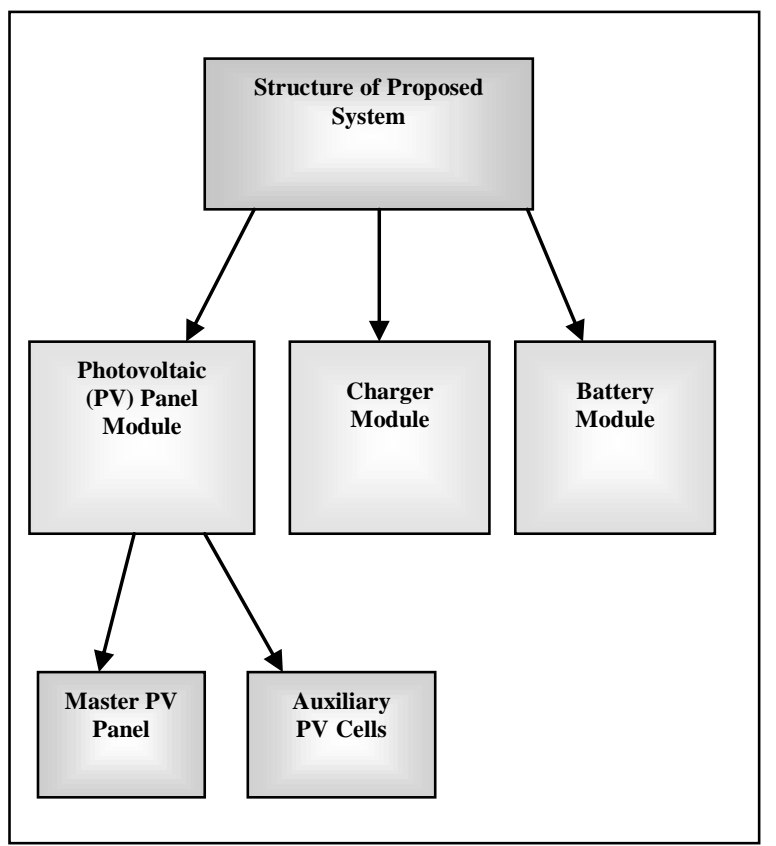

Fig. (3): Structure of the proposed PV System

$\underline{T h i r d l y}$, the proposed neural network is trained with multiple types of faults for the PV parts (panel, charger and battery).

Fourthly, the neural network begins the diagnostic process as described in fig. (4). The tasks of the diagnostic operation in the following steps: 


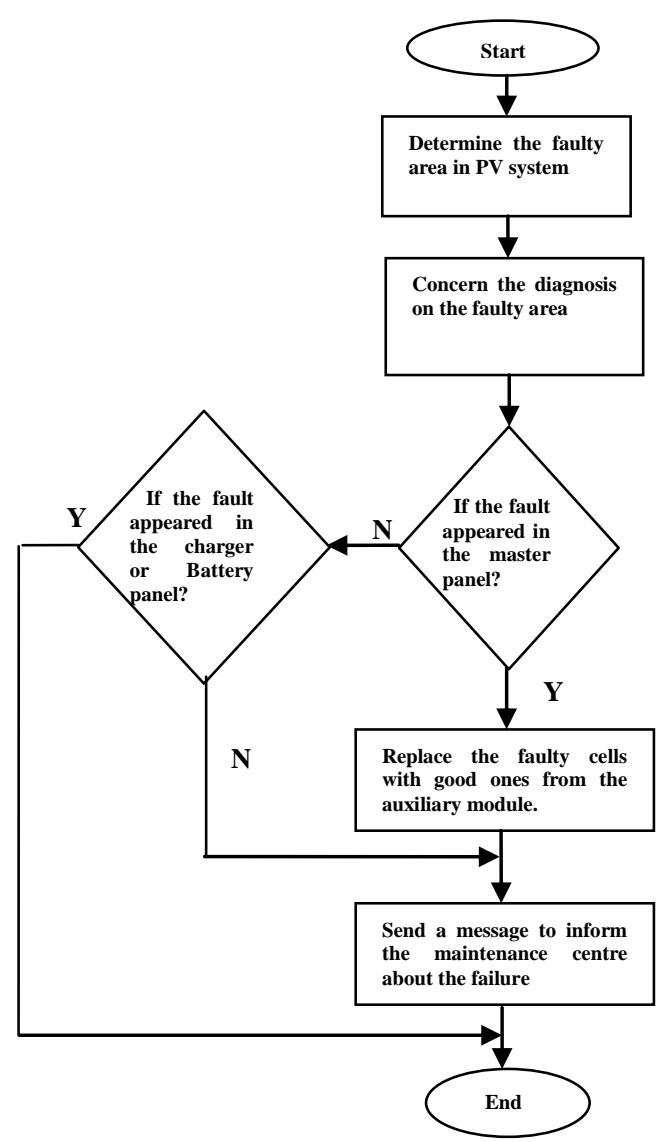

Fig. (4): The diagnostic operation of the proposed system

(1) Detecting the failure operation of the faulty module(s), the proposed system determines which area (group of PV cells) has the failure

(2) The system concerns the diagnostic process for the faulty module(s) or group(s) of PV cells only rather than diagnosis the whole PV system.

(3) If the proposed system diagnoses faulty cells in the master PV panel. These cells are replaced with correct ones from the auxiliary cells automatically online.

(4) The proposed system sends a message for the maintenance centre to inform about the faulty cells now

in the auxiliary cells. Then, the centre can repair them without affecting the operation of the load.

(5) If the failure appeared in the charger or the battery module, the algorithm sends also alarmed message for the maintenance centre for repairing.

The implementation of the suggested diagnostic system is described as:

1- It is implemented under the environment of the Matlab/Simulink.

2- The proposed neural network has used back propagation algorithm that proved its success for the diagnostic systems.
3- The optimum topology of the ANN obtained from the results of the genetic algorithm is: It has two hidden layers with 24 neurons.

\section{APPLICABILITY OF THE PROPOSED DIAGNOSTIC SYSTEM AND ITS RESULTS}

The proposed system is a general purpose diagnostic PV system. It can be used for diagnosis different PV systems. It has been applied to diagnose the faults of a PV system used to feed the power for a bulletin board in the high way used for traffic's instructions as a case of study.

The proposed neural network is trained for 8-types of the faults for each part. The output number 0 represents the PV module in normal state. While, the output numbers (1-to-5) deal with the faults appeared in the master PV panel. Where, O/P1: represents the degradation state in $\mathrm{PV}$ module, $\mathrm{O} / \mathrm{P} 2$ : represents the short-circuit state in $\mathrm{PV}$ module, $\mathrm{O} / \mathrm{P}$ 3: represents the open-circuit state in $\mathrm{PV}$ module, O/P4: represents the degradation of power state, O/P5: represents the partial shading in PV master panel module. O/P6: represents the charger failure state, $\mathrm{O} / \mathrm{P} 7$ represents the battery failure state. Table (1): represents a comparison between the simulated results obtained from a fuzzy system [9], traditional neural network system without optimizing its topology [10] and the proposed diagnostic system. While, figures (4-7) are represented comparisons between the results obtained from the three systems at different temperatures and irradiations. It is found that, the proposed system has proved its significant performance compared with the other systems. However, the proposed system can be used for diagnosis different types of real-time PV systems in good manner. Therefore, the performance of these systems can be improved.

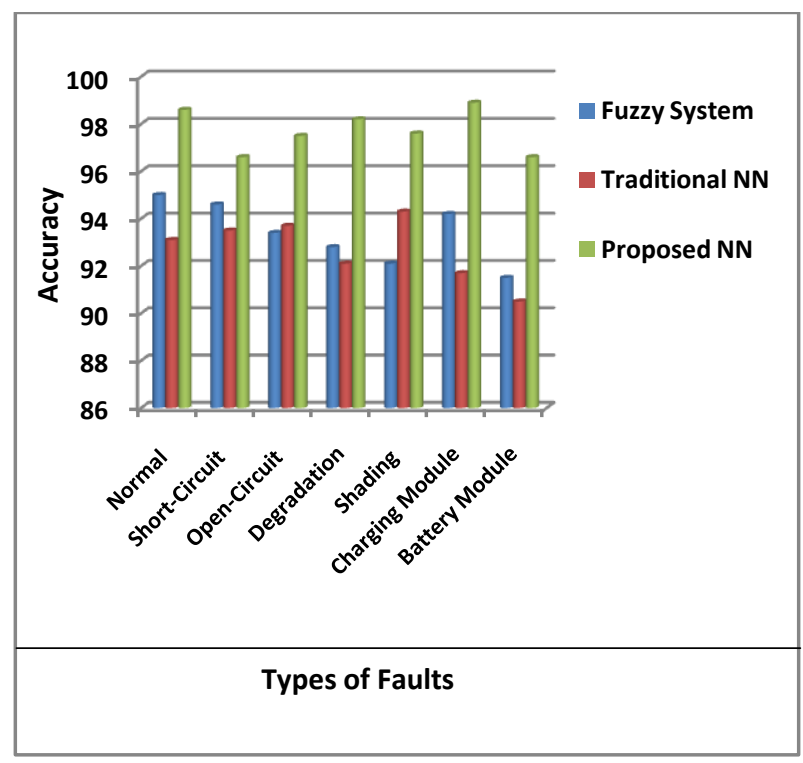

Fig. (4): A comparison between the accuracy of the fuzzy System, Traditional NN and the proposed NN systems at

Temperature $=33{ }^{\circ} \mathrm{C}$, and Irradiation $380\left(\mathrm{~W} / \mathrm{m}^{2}\right)$ applied for a bulletin board in the high way. 
Table (1): A comparison between the simulated results obtained from a fuzzy system, traditional neural network system and the proposed system.

\begin{tabular}{|c|c|c|c|c|c|c|c|c|}
\hline \multicolumn{3}{|c|}{ Environment Conditions } & \multicolumn{6}{|c|}{ Simulation Results } \\
\hline \multirow{2}{*}{$\begin{array}{l}\text { Type } \\
\text { faults }\end{array}$} & \multirow{2}{*}{$\begin{array}{l}\text { Temper- } \\
\text { ature }{ }^{\circ} \mathrm{C}\end{array}$} & \multirow{2}{*}{$\begin{array}{l}\text { Irradia- } \\
\text { tion } \\
\left(\mathrm{W} / \mathbf{m}^{2}\right)\end{array}$} & \multicolumn{2}{|c|}{ Fuzzy System } & \multicolumn{2}{|c|}{ Traditional NN System } & \multicolumn{2}{|c|}{ Proposed NN System } \\
\hline & & & Accuracy & $\begin{array}{l}\text { Time } \\
\text { (sec.) }\end{array}$ & Accuracy & $\begin{array}{l}\text { Time } \\
\text { (sec.) }\end{array}$ & Accuracy & $\begin{array}{l}\text { Time } \\
\text { (sec.) }\end{array}$ \\
\hline \multirow[t]{2}{*}{ Normal } & 33 & 380 & 95.0 & 9.7 & 93.1 & 8.8 & 98.6 & 5.4 \\
\hline & 35 & 570 & 94.7 & 9.5 & 93.5 & 8.7 & 97.7 & 5.3 \\
\hline \multirow[t]{2}{*}{ Short-Circuit } & 33 & 380 & 94.6 & 9.4 & 93.5 & 8.6 & 96.6 & 4.4 \\
\hline & 35 & 570 & 94.1 & 9.3 & 93.9 & 8.3 & 96.2 & 6.3 \\
\hline \multirow[t]{2}{*}{ Open-Circuit } & 33 & 380 & 93.4 & 9.1 & 93.7 & 8.1 & 97.5 & 6.9 \\
\hline & 35 & 570 & 93.6 & 10.1 & 92.5 & 7.9 & 97.8 & 5.8 \\
\hline \multirow[t]{2}{*}{ Degradation } & 33 & 380 & 92.8 & 9.8 & 92.1 & 10.7 & 98.2 & 5.6 \\
\hline & 35 & 570 & 92.4 & 9.2 & 92.3 & 10.5 & 98.1 & 4.8 \\
\hline \multirow[t]{2}{*}{ Shading } & 33 & 380 & 92.1 & 8.9 & 94.3 & 11.6 & 97.6 & 5.7 \\
\hline & 35 & 570 & 92.2 & 8.7 & 92.9 & 11.3 & 97.8 & 3.9 \\
\hline \multirow{2}{*}{$\begin{array}{l}\text { Charging } \\
\text { Module }\end{array}$} & 33 & 380 & 94.2 & 10.2 & 91.7 & 9.5 & 98.9 & 3.7 \\
\hline & 35 & 570 & 94.6 & 10.5 & 91.6 & 9.7 & 98.6 & 3.5 \\
\hline \multirow{2}{*}{$\begin{array}{l}\text { Battery } \\
\text { Module }\end{array}$} & 33 & 380 & 91.5 & 8.8 & 90.5 & 10.8 & 96.6 & 4.7 \\
\hline & 35 & 570 & 91.2 & 8.5 & 90.2 & 10.5 & 96.6 & 4.6 \\
\hline
\end{tabular}

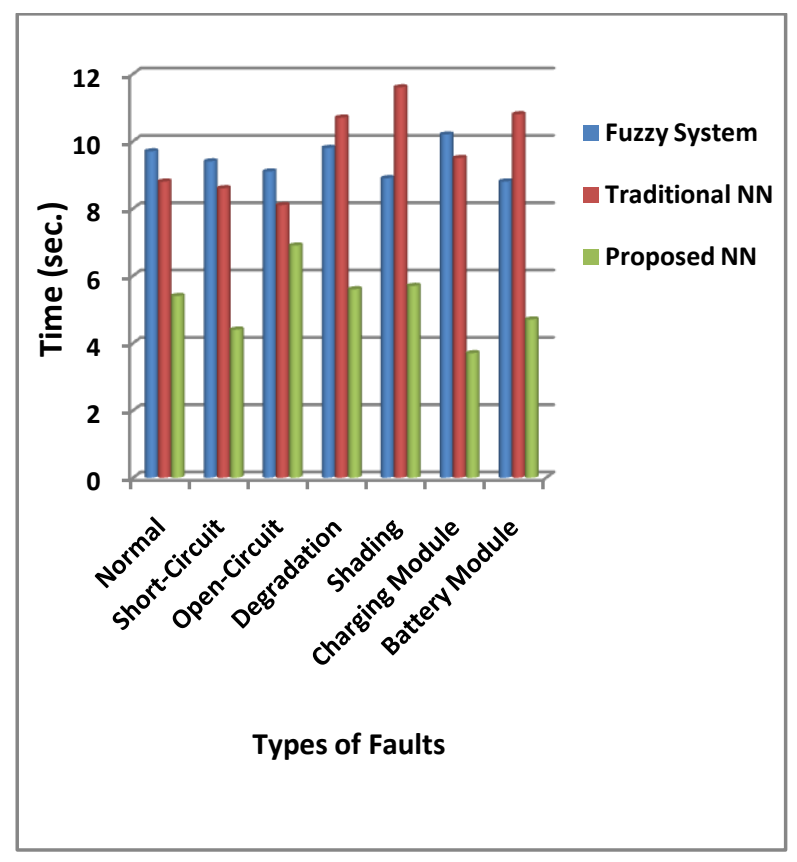

Fig. (5): A comparison between the time of the fuzzy System, Traditional NN and the proposed NN systems at Temperature $=33^{\circ} \mathrm{C}$, and Irradiation $380\left(\mathrm{~W} / \mathrm{m}^{2}\right)$ applied for a bulletin board in the high way.

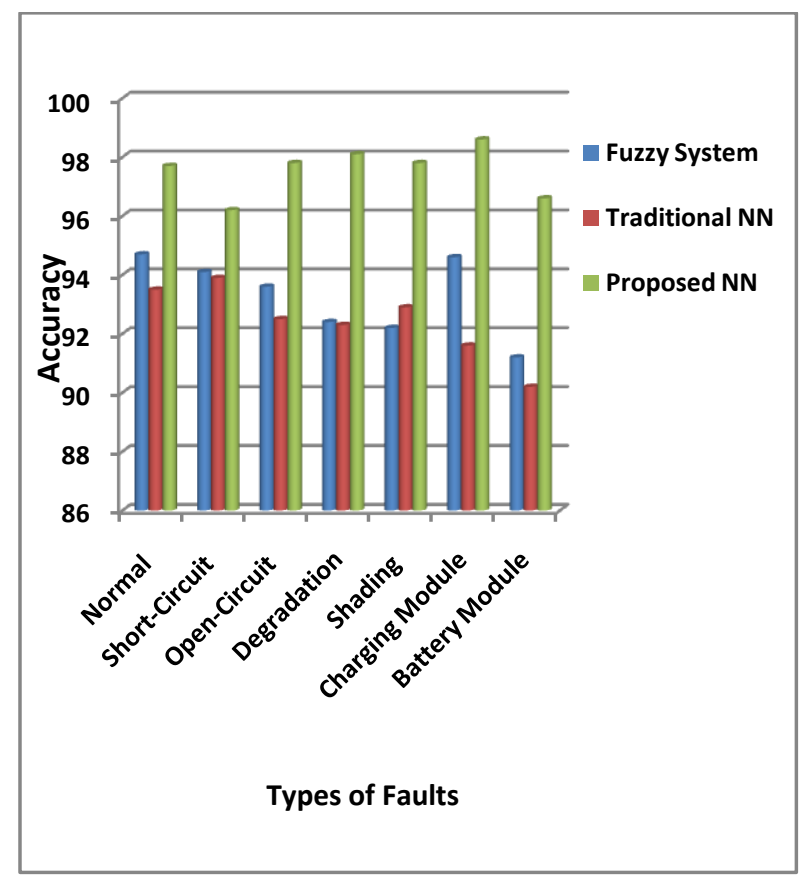

Fig. (6): A comparison between the accuracy of the fuzzy System, Traditional NN and the proposed NN systems at Temperature $=35^{\circ} \mathrm{C}$, and Irradiation $570\left(\mathrm{~W} / \mathrm{m}^{2}\right)$ applied for a bulletin board in the high way. 


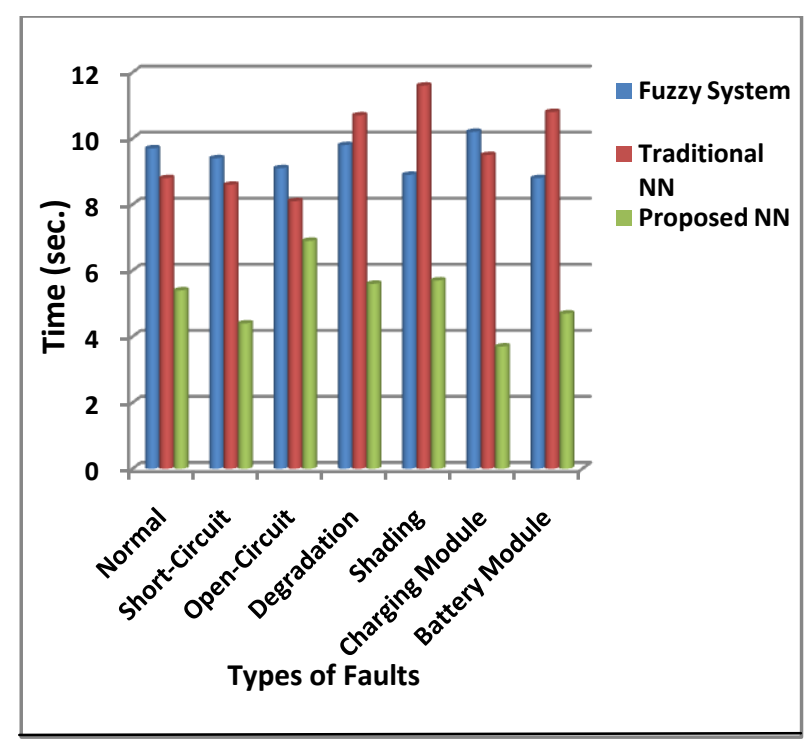

Fig. (7): A comparison between the time of the fuzzy System, Traditional NN and the proposed NN systems at Temperature $=35{ }^{\circ} \mathrm{C}$, and Irradiation $570\left(\mathrm{~W} / \mathrm{m}^{2}\right)$ applied for a bulletin board in the high way.

\section{CONCLUSION}

Neural networks have proved their success in solving the diagnostic problems. The proposed system is a new member in the neural network diagnostic families. It uses the genetic algorithm to optimize its topology. It introduces the diagnosis and repairing the PV systems. The suggested system can improve the performance of the diagnostic systems due to the following features:

1- The proposed system introduces a new complete algorithm for diagnosis and repairing the PV systems.

2- It can improve the performance of the traditional neural network system by optimizing its topology using the genetic algorithm technique.

3- It can diagnose multiple faults in parallel and so speed-up the diagnostic process.

4- The system can replace the faulty cell(s) with good-operated ones online. So, it can repair the failure immediately and on-line.

5- It can decrease the diagnostic time and speed-up the process.

6- It can be used for the modern complex PV systems that are considered one of the main issues of modern technology.

7- It can divide the PV panel into group of sub-areas. And so, the diagnostic process into two steps. The first one deals with detecting the failure and the second one concerns with diagnosis the cause for the failed area only.

However, the proposed system has been applied for the different types of PV systems. Its obtained results are compared with the results for fuzzy and traditional neural network-based diagnostic systems. These results have proved its increasing for the accuracy and decreasing the time for the diagnostic process.

\section{REFERENCES}

[1] K. H. Chao, C. J. Li, and S. H. Ho, 2008 , "Modeling and fault simulation of photovoltaic generation systems using circuit-based model," Proc. of IEEE Int'l Conf. on Sustainable Energy Technologies.

[2] Y. Kim, N. Chang, Y. Wang, and M. Pedram, 2010, "Maximum power transfer tracking for a photovoltaicsupercapacitor energy system, "Proc. of the Int'l Symposium on Low Power Electronics and Design, pp. $201-$.

[3] M. Davarifar, A. Rabhi, A. El-Hajjaji, J. Bosche, X. Pierre, 2013, Improved Real Time Amorphous PV Model for Fault Diagnostic Usage, in: A. Hakansson, M. Höjer, R.J. Howlett, L.C. Jain (Eds.), Sustainability in Energy and Buildings, Springer Berlin Heidelberg, pp. 179-188.

[4] S.K. Firth, K.J. Lomas, S.J. Rees, (2010), A simple model of PV system performance and its use in fault detection, Solar Energy, 84, pp. 624-635.

[5] T. Takashima, J. Yamaguchi, K. Otani, T. Oozeki, K. Kato, M. Ishida, 2009, Experimental studies of fault location in PV module strings, Solar Energy Materials and Solar Cells, Vol. 93, pp. 1079-1082.

[6] A. Chouder, S. Silvestre, 2010, Automatic supervision and fault detection of PV systems based on power losses analysis, Energy Conversion and Management, Vol. 51, pp. 1929-1937.

[7] Z. Ye, Y. Ling, B. Lehman, J. de Palma, J. Mosesian, R. Lyons, 2012, Decision tree-based fault detection and classification in solar photovoltaic arrays, in: Applied Power Electronics Conference and Exposition (APEC), 2012 Twenty-Seventh Annual IEEE, pp. 93-99.

[8] L. Schirone, F. P. Califano, U. Moschella, U. Roccs, 2013, "Fault finding in a 1 MW photovoltaic plant by reflectometry," IEEE Conf. on Photovoltaic Energy Conversion, pp.204-208.

[9] D. Riley, J. Johnson, 2012, Photovoltaic prognostics and heath management using learning algorithms, in: Photovoltaic Specialists Conference (PVSC), 2012, 38 IEEE, pp. 1535-1539.

[10] P. Ducange, M. Fazzolari, B. Lazzerini, F. Marcelloni, 2011, An intelligent system for detecting faults in photovoltaic fields, in: Intelligent Systems Design and Applications (ISDA), 2011, $11^{\mathrm{th}}$ International Conference, pp. 1341-1346.

[11] Luca Bonsignore, Mehrdad Davarifar, Abdelhamid Rabhi, Giuseppe M.Tina and Ahmed Elhajjaji, 2014, NeuroFuzzy fault detection method for photovoltaic systems, Energy Procedia Vol. 62, pp. 431 - 441

[12] A. Coleman, J. Zalewski, 2011, Intelligent fault detection and diagnostics in solar plants, in: Intelligent Data Acquisition and Advanced Computing Systems (IDAACS), 2011 IEEE 6th International Conference on, IEEE, pp. 948-953.

[13] M. Davarifar, A. Rabhi, A. El Hajjaji, (2013), Comprehensive Modulation and Classification of Faults and Analysis Their Effect in DC Side of Photovoltaic System, Energy and Power Engineering, Vol. 5 pp. 230237. 
[14] M. Davarifar, A. Rabhi, A. El-Hajjaji, M. Dahmane, 2013, Real-time Model base Fault Diagnosis of Photovoltaic Panels Using Statistical Signal Processing, in: International Conference on Renewable Energy Research and Applications (ICRERA2013), Madrid, Spain.

[15] M. Davarifar, A. Rabhi, A. El Hajjaji, M. Dahmane, 2013, New method for fault detection of PV panels in domestic applications, in: Systems and Control (ICSC), 2013 3rd International Conference, pp. 727-732.

[16] H. H. Lee, L. M. Phuong, P. Q. Dzung, N. T. Dan Vu, and L. D. Khoa, 2010, "The new maximum power point tracking algorithm using ANN-based solar PV systems," in Proceedings of the IEEE Region 10 Conference (TENCON '10), Fukuoka, Japan, pp. 2179-2184.

[17] Oleksiy Roshchupkin, Radislav Smid, Volodymyr Kochan, Anatoly Sachenko, 2013, Multisensors Signal Processing Using Microcontroller and Neural Networks Identification. Sensors \& Transducers Journal, Vol.24, No.8, pp. 1-6.
[18] Turchenko I. , 2007, Accurate Recognition of MultiSensor Output Signal Using Modular Neural Networks / Turchenko I., Kochan V., Sachenko A., International Journal of Information Technology and Intelligent Computing, Vol. 2, No. 1, pp. 27- 47.

[19] Tomoiagă B, Chindriş M, Sumper A, Sudria-Andreu A, Villafafila-Robles R. Pareto, 2013, Optimal Reconfiguration of Power Distribution Systems Using a Genetic Algorithm Based on NSGA-II. Energies, Vol. 6 , No. 3, pp. 1439-1455.

[20] M. Namdari, H. Jazayeri-Rad, and S.-J. Hashemi, "Process fault diagnosis using support vector machines with a genetic algorithm based parameter tuning," Journal of Automation and Control, vol. 2, no. 1, pp. 1-7, 2014.

[21] R. Cretulescu, D. Morariu, M. Breazu, and L. Vintan, 2012, "Weights space exploration using genetic algorithms for meta-classifier in text document classification," Studies in Informatics and Control, Vol. 21, No. 2, pp. 147-154. 